\title{
MISCEL·LÀNIA
}

\section{STUDIO PRELIMINARE SULLA COMMITTENZA ARTISTICA FEMMINILE NELLA SARDEGNA TRA X E XI SECOLO ${ }^{1}$}

\section{PRELIMINARY STUDY ABOUT FEMALE ARTISTIC PATRONAGE IN SARDINIA BETWEEN THE $10^{\mathrm{TH}}$ AND $11^{\mathrm{TH}}$ CENTURY}

\author{
Valeria Carta \\ Università degli Studi di Cagliari
}

\begin{abstract}
Resumen: A Sardenya són molts els fragments medievals recuperats a les esglésies actualment destruïdes i sovint reconeguts com fora de context. Per tant, als especialistes els és difícil investigar adequadament artefactes com els epígrafs greco-sards. Alguns d'aquests informen dels noms d'homes i dones que probablement formaven part de les famílies aristocràtiques que governaven l'illa. La comparació d'evidències sardes amb les trobades al sud d'Itàlia podria aportar respostes sobre aquest període. A més, pot ser significativament eficaç per revelar totes les connexions entre el mecenatge artístic i les dones a Sardenya.
\end{abstract}

Palabras clave: Sardenya, edat mitjana, mecenatge artístic, comissió de dones, epígrafs.

Abstract: In Sardinia many are the medieval fragments recovered in currently destroyed churches and frequently recognized as out of context. Therefore, specialists find it difficult to proper investigate artefacts such as the Greek-Sardinians epigraphs. Some of those report the men and women names who probably were part of the aristocratic families that ruled the island. Comparing Sardinian evidences with the ones found in south Italy could allegedly provide responses about this period. Moreover, it might be significantly effective in order to reveal all the connections between artistic patronage and women in Sardinia.

Keywords: Sardinia, Middle Ages, artistic patronage, women commission, epigraphs.

\footnotetext{
${ }^{1}$ La presente pubblicazione è stata prodotta durante la frequenza del corso di dottorato in Storia, Beni Culturali e Studi Internazionali dell'Università degli Studi di Cagliari, XXXV ciclo, con il supporto di una borsa di studio finanziata con le risorse del P.O.R. Sardegna F.S.E. 2014-2020 - Asse III «Istruzione e Formazione» - Obiettivo Tematico 10, Priorità d'investimento 10ii), Obiettivo Specifico 10.5, Azione dell'accordo di Partenariato 10.5.12.
} 


\section{Introduzione}

La produzione artistica nella Sardegna tra $\mathrm{X}$ e XI secolo è giunta a noi perlopiù allo stato di frammento. ${ }^{2}$ Nella maggior parte dei casi, si conservano manufatti riconducibili ad elementi di arredo liturgico, ${ }^{3}$ la cui committenza presenta interessanti spunti di riflessione. Questi pezzi scultorei si inseriscono cronologicamente in un'epoca storica che per l'Isola lamenta l'assenza di fonti scritte e non offre pertanto materiali sufficienti per ricostruirne la situazione politica. ${ }^{4}$ Il momento di passaggio tra la fine della dominazione bizantina e la successiva quadripartizione del territorio in giudicati $o$ regni, come riportano le fonti, sono aspetti ancora non del tutto chiari. ${ }^{5}$ Il primo documento che testimonia l'esistenza di quattro giudici aventi pari dignità e distinti ambiti territoriali di governo è una lettera di Gregorio VII, redatta a Capua il 14 ottobre 1073.6 Nella missiva il pontefice redarguiva i quattro sovrani sardi, Orzocco di Calàri, Orzocco di Arborea, Mariano di Torres e Costantino di Gallura, affinché rispettassero

\footnotetext{
2 L'architettura databile a questo periodo conta diversi esempi ancora fortemente problematici e di difficile lettura critica. Cfr. Roberto Coroneo, Scultura mediobizantina in Sardegna. Nuoro: Poliedro, 2000, con bibliografia precedente.

3 Andrea Pala, Arredo liturgico medievale. La documentazione scritta e materiale in Sardegna fra IV e XIV secolo. Cagliari: Edizioni AV, 2011, p. 33-36.

4 Andrea Pala - Nicoletta Usai, «Manufatti scultorei dai rinvenimenti marini in area costiera della Sardegna (VI-X secolo)», Rossana Martorelli (ed.), Know the sea to live the sea. Conoscere il mare per vivere il mare. Perugia: Morlacchi Editore, 2019, p. 296; Corrado Zedda - Raimondo Pinna, «La nascita dei giudicati. Proposta dello scioglimento di un enigma storiografico", Archivio Storico Giuridico di Sassari 12 (2007), p. 74.

5 Salvatore Cosentino, «Potere e istituzioni nella Sardegna bizantina», Paola Corrias - Salvatore Cosentino (coords.), Ai confini dell'Impero: storia, arte e archeologia della Sardegna bizantina. Cagliari: M\&T Sardegna, 2002, p. 11.

6 Pasquale Tola, Codice Diplomatico della Sardegna, vol. I. Sassari: Carlo Delfino Editore, 1984, p. 156.
}

l'ortodossia cattolica. ${ }^{7}$ La stabilizzazione del nuovo assetto politico, dunque, si può far risalire con sicurezza solo agli inizi dell'XI secolo. La fase storica che precede la quadripartizione dell'isola viene convenzionalmente definita «bizantina», terminologia che, impiegata nell'ambito della cultura artistica della Sardegna, inquadra un arco cronologico che procede dal VI al IX secolo, periodo durante il quale l'Isola sarebbe stata sotto il controllo politico e amministrativo dell'impero di Costantinopoli. ${ }^{8}$ Non sono però ancora chiare le modalità e i tempi del distacco da Bisanzio. Allo stato degli studiè difficile ricostruire gli accadimenti che favorirono l'uscita della Sardegna dall'orbita imperiale e ne determinarono il nuovo assetto politico.9 Sono invece numerosi gli indizi che testimoniano una permanenza di rapporti e legami con il mondo greco-orientale ancora nel corso del IX e X secolo. ${ }^{10} \mathrm{E}$ forse utile aggiungere a questa sintesi storica le riflessioni fatte più recentemente da Michele Orrù. Lo studioso esamina le fonti greche di età bizantina in Sardegna tra il VI e il XII secolo, proponendo per questo stesso periodo l'utilizzo della terminologia «influsso bizantino», a suo avviso preferibile rispetto alla definizione ancora presente di «Sardegna bizantina». Orrù è portato a credere che l'Isola possa essere considerata propriamente bizantina solo fino al 751, anno della caduta dell'Esarcato d'Africa, o poco tempo dopo, quando sembra che la zecca di Cartagine sia stata spostata a Cagliari. ${ }^{11}$

7 Luciano Gallinari «Il giudicato di Calari tra XI e XIII secolo. Proposte di interpretazioni istituzionali», RiMe. Rivista dell'Istituto di Storia dell'Europa Mediterranea 5 (2010), p. 154.

8 Roberto Coroneo, «La cultura artistica», Paola Corrias Salvatore Cosentino (coords.), Ai confini dell'Impero. Storia, arte e archeologia della Sardegna bizantina. Cagliari: M\&T Sardegna, 2002, p. 99.

9 Olivetta Schena, La Sardegna Medievale nel contesto italiano e mediterraneo (secc. XI-XV). Noceto: Monduzzi Editoriale S.r.1., 2011, p. 9.

${ }^{10}$ Ibid., p. 9.

${ }^{11}$ Michele Orrù, Le fonti greche di età bizantina per lo studio della Sardegna altomedievale (VI-XII secolo). PhD Thesis, Università degli Studi di Cagliari, 2012-2013, p. 2223. 


\section{I frammenti epigrafici d'arredo liturgico}

Tra le testimonianze più rilevanti di questo momento storico si prendano in esame le epigrafi medioelleniche rinvenute nel solo territorio che corrispondeva al Giudicato di Cagliari. ${ }^{12}$ Si tratta di sei esemplari scultorei ritrovati prevalentemente in contesti ecclesiastici presumibilmente di matrice orientale, associati ad altri numerosi frammenti litici. ${ }^{13}$ La maggior parte di questi giunge da contesti profondamente modificati rispetto alla rispettiva conformazione originaria. Si tratta per lo più di chiese allo stato di rudere o completamente scomparse o, ancora, di contesti architettonici che nel corso dei secoli hanno subito profonde e definitive modiche sia in termini di impianto che di arredo liturgico. È difficile affermare con certezza se questi materiali siano stati ideati per i contesti a cui vengono generalmente riferiti dalla storiografia. ${ }^{14}$ Certamente la maggior parte di questi elementi marmorei risultano ormai fuori contesto, ${ }^{15}$ mentre una larga parte è stata riutilizzata per esigenze diverse su strutture posteriori. ${ }^{16}$ I manufatti scultorei, nel complesso, sembrerebbero essere il frutto dell'esigenza rappresentativa di una committenza aulica in cerca di affermazione e prestigio. ${ }^{17}$ Oltre a essere gli unici documenti materiali coevi, le epigrafi rappresentano il marcatore culturale di una classe sociale che si esprime mediante una tecnica elevata e raffinata, quali produzioni, forse locali, commissionate dai giudici. Convenzionalmente, infatti, i nomi tramandati dagli elementi di arredo liturgico rivenuti sul territorio si riferirebbero a quelli dei primi sovrani del Giudicato di Cagliari. ${ }^{18}$

\footnotetext{
12 Ibid., p. 25, 44.

13 Roberto Coroneo, Scultura mediobizantina in..., op. cit., p.7.

${ }^{14}$ Ibid., p. 37-38.

15 Roberto Coroneo, Arte in Sardegna dal IV alla metà dell’XI secolo. Cagliari: Edizioni AV, 2011, p. 397.

16 Valeria Carta, «I marmi mediobizantini reimpiegati nelle chiese sarde in area costiera», Rossana Martorelli (ed.), Know the sea to live the sea. Conoscere il mare per vivere il mare. Perugia: Morlacchi Editore, 2019, p. 342.

17 Roberto Coroneo, La cultura artistica..., op. cit., p. 100-101.

18 Roberto Coroneo, Scultura mediobizantina in..., op. cit., p. 31.
}

\section{I primi studi}

Già nel 1631 J.F. Carmona nel Alabancas de los Santos de Sardena documentava la presenza di una di queste epigrafi, nello specifico di una di quelle attualmente custodite ad Assemini, centro abitato poco distante da Cagliari. ${ }^{19}$ Nella seconda metà dell'Ottocento fiorirono una serie di ricerche sulla natura epigrafica e linguistica condotte da eruditi e studiosi, come Giovanni Spano (1857), Antonio della Marmora (1860), Celestino Cavedoni (1860) e Pietro Martini (1861). Un'edizione organica di tutte le iscrizioni fu però elaborata solo da Antonio Taramelli (1906) attraverso una serie di contributi poi confluiti in un unico articolo pubblicato in Archivio Storico Sardo. ${ }^{20}$ A uno studio più strettamente storico-artistico sono dedicati i contributi della seconda metà del Novecento. Tra i primi quelli di Raffaello Delogu (1953), seguiti da quelli di Renata Serra (1970) e Letizia Pani Ermini (1981). ${ }^{21}$ I più recenti scritti in materia sono, invece, riconducibili a Roberto Coroneo. $^{22}$ Gli apporti alla ricerca forniti da questi studiosi hanno prediletto un approccio atto a sottolineare l'alto livello tecnico dei manufatti e le loro caratteristiche stilistiche e formali. Questi lavori si sono concentrati sui caratteri descrittivi dei manufatti, sulle particolarità linguistiche legate al lessico utilizzato e ai titoli onorifici, nonché sull'individuazione delle possibili aree di influenza artistica e di produzione. ${ }^{23}$

Per quanto concerne gli aspetti legati alla committenza, la riflessione appare complessa. I ricercatori che si sono occupati del problema si

\footnotetext{
19 Ibid., p. 7.

${ }^{20}$ Ibid., p. 7.

${ }^{21}$ Ibid., p. 8.

${ }^{22}$ Roberto Coroneo, Scultura mediobizantina in..., op. cit.; Roberto Coroneo, Arte in Sardegna..., op. cit.

${ }^{23}$ Roberto Coroneo, Scultura mediobizantina in..., op. cit., p. 23-30 e 107-206. Guglielmo Cavallo, «Le tipologie della cultura nel riflesso delle testimonianze scritte», Centro italiano di studi sull' alto medioevo, Bisanzio, Roma e l'Italia nell'alto Medioevo. 3-9 aprile 1986, vol. II. Spoleto: Centro italiano di studi sull' alto medioevo, 1988, p. 467-516; Giulio Paulis, Lingua e cultura nella Sardegna bizantina. Testimonianze linguistiche dell'influsso greco. Sassari: L'Asfodelo, 1983.
} 
sono, per lo più, concentrati sull'individuazione dei personaggi, intentando un'identificazione che, priva di un supporto documentario, appare piuttosto incerta. ${ }^{24}$ Un nuovo esame critico potrebbe dare spunti di riflessione basati su altri aspetti. Uno di questi riguarda le modalità con le quali il committente si pone in evidenza, si mostra, all'interno di un edificio sacro per lasciare traccia della sua persona vivente. La sua attività in questa prospettiva è quella di marcatore del ruolo sociale espresso attraverso l'opera d'arte. ${ }^{25}$ Il committente è il promotore dell'esecuzione di un'opera e andrebbe preferibilmente distinto dal donatore, ossia da colui che finanzia l'opera stessa. ${ }^{26} \mathrm{Il}$ caso delle epigrafi greche del Giudicato di Cagliari amplia gli orizzonti della ricerca su un ulteriore aspetto connesso alla committenza, nello specifico a quella femminile, lasciato apparentemente in ombra. Il ribaltamento della prospettiva di genere, dal maschile al femminile, in Sardegna offre la possibilità di un'indagine specifica che vada oltre la semplice identificazione del personaggio citato. D'altro canto, gli interrogativi che si aprono sono molteplici. Infatti, anche se le figure femminili nelle epigrafi sono indicate accanto a quelle maschili, le difficoltà messe in luce precedentemente permangono. $\mathrm{Nel}$ caso specifico, inoltre si aggiunga il fatto che il ruolo della donna nell'Isola non è ancora emerso nella sua totalità. Dunque non appare sufficiente chiedersi solo chi siano queste donne, quanto piuttosto sarebbe proficuo riflettere sul ruolo che esse hanno ricoperto nella committenza di taluni arrendi liturgici, se non addirittura di interi edifici di culto. L'ultimo studio completo in ordine di tempo sul tema è quello di Roberto Coroneo, che nella monografia Scultura Mediobizantina in Sardegna edito da Poliedro nel 2000, riassume tutti i tratti comuni riconosciuti a queste

\footnotetext{
${ }^{24}$ Roberto Coroneo, Scultura mediobizantina in..., op. cit., p. 31-36.

25 B. Brenk, «Committenza», Enciclopedia dell'Arte Medievale. Roma: Istituto della Enciclopedia Italiana, 1994, p. 203.

26 IIbid., p. 203.
}

epigrafi, all'interno di un più ampio quadro della scultura sarda. Lo studioso anticipa anche alcuni possibili percorsi di riflessione, sottolineando la stretta vicinanza tra il materiale lapideo isolano, composto in gran parte oramai di frammenti, e le opposte sponde della Campania. ${ }^{27}$

\section{Manufatti con epigrafi e nomi femminili: un problema aperto}

Tra il materiale epigrafico sono cinque gli epitaffi che riportano nomi femminili o presunti tali. Lo studio, se pur ancora in fase preliminare, ha preso avvio dall'analisi della letteratura critica. L'esame del materiale, gran parte riconducibile al secolo scorso, ha evidenziato alcune discrepanze tra la situazione descritta e quella odierna. Nello specifico, appare dubbia oggi la collocazione di alcuni dei materiali, come quelli ricordati dalla bibliografia, nei magazzini del Museo Archeologico Nazionale di Cagliari. ${ }^{28} \mathrm{Si}$ auspica dunque la possibilità di effettuare una ricerca sul campo nei suddetti depositi, funzionale a confermare o smentire la presenza dei materiali riconducibili ai contesti sopraindicati. Secondo tale previsione sarebbe vantaggioso operare una nuova campagna fotografica che completi i documenti a disposizione. Successivamente all'analisi del materiale locale, appare utile la disamina delle evidenze epigrafiche presenti in altri territori, a partire da quello nazionale. Dall'analisi delle pubblicazioni del Centro Italiano di Studi sull'Alto Medioevo potrebbero emergere interessanti parallelismi con altre realtà del Sud Italia, oltre a quelle già evidenziante in ambito campano. ${ }^{29}$ La koinè mediterranea entro la quale si muovono questi manufatti costituisce un interessante elemento di riflessione che comporta un allargamento del raggio di indagine verso i territori orientali dai quali potrebbero avere attinto

\footnotetext{
27 Roberto Coroneo, Scultura mediobizantina in..., op. cit., p. 148-177.

28 Ibid., p. 216-217.

${ }^{29}$ Ibid., p. 148-177.
} 
usi e costumi anche i sovrani sardi. Sarebbe utile individuare elementi comuni che possano aiutare a determinare l'attività in campo artistico delle figure femminili che tuttavia, almeno in questa fase, sembrerebbe già orientata verso elementi di arredo liturgico. ${ }^{30} \mathrm{Si}$ è in parte già provveduto all'esame dei documenti scritti che, tuttavia, per questo periodo risultano numericamente limitati. La raccolta di Pasquale Tola, pubblicata nel Codex Diplomaticus Sardiniae (1861 e 1868), non conserva alcuna testimonianza riconducibile al periodo in questione. I personaggi citati nelle epigrafi isolane non sembrerebbero coincidere con quelli riportati dai primi documenti sardi che, è bene ricordarlo, sono databili ai primi anni dell'XI secolo.

\section{L'iscrizione di Unuspiti e Sorica}

Seguendo l'impostazione cronologica proposta da Roberto Coroneo, l'iscrizione più antica, fra le sei rinvenute, è quella proveniente dalle rovine della chiesa di Santa Sofia, nel territorio tra i centri di Decimoputzu e Villasor, in provincia di Cagliari ${ }^{31}$ (fig. 1-2). I marmi furono prelevati nelle adiacenze della struttura ecclesiastica poco prima del $1860 .^{32}$ L'ultima collocazione segnalata è quella dei depositi del museo Archeologico Nazionale di Cagliari. Il manufatto litico, che originariamente si presentava come un unico pezzo, fu rinvenuto scisso in due parti, entrambe in forma di parallelepipedo, di colore bianco e aventi all'incirca la stessa misura

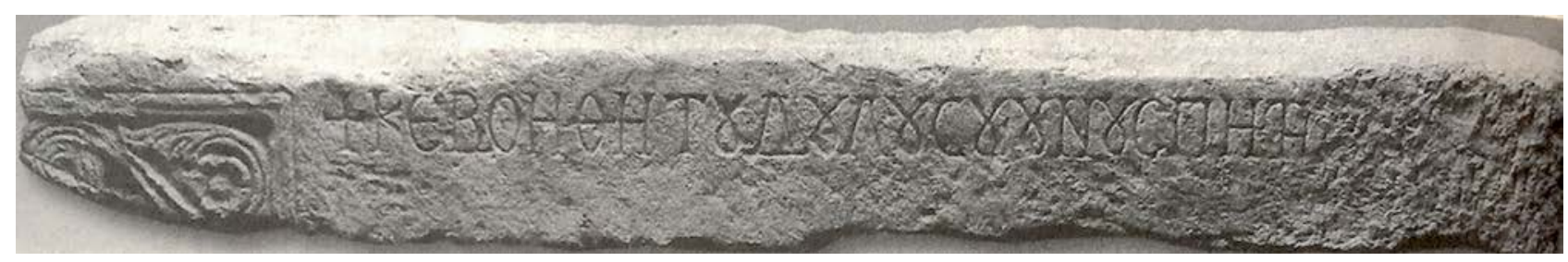

Figura 1. Cagliari, epigrafe di Unuspiti e Sorica (da Coroneo 2000).

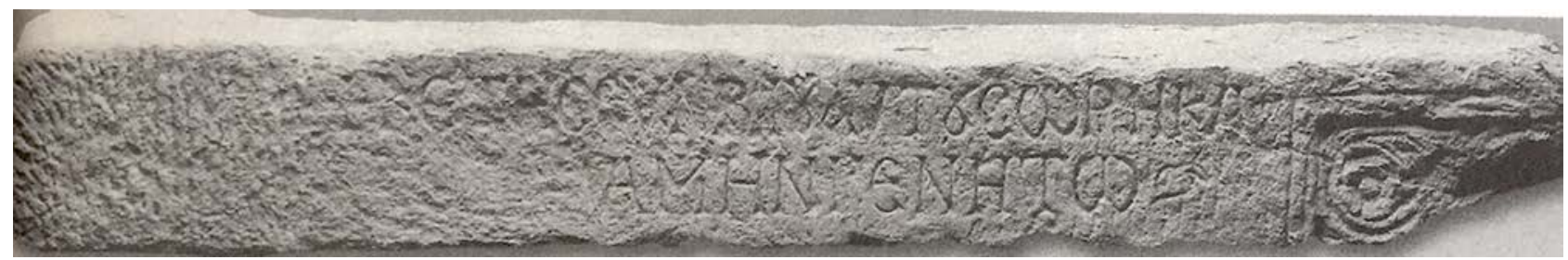

Figura 2. Cagliari, epigrafe di Unuspiti e Sorica (da Coroneo 2000).

${ }^{30}$ Ibid., p. 32.

\footnotetext{
${ }^{31}$ Antonio Taramelli, «Di alcuni monumenti epigrafici bizantini», Archivio Storico Sardo III (1907), p. 79-81. Roberto Coroneo, Scultura mediobizantina in..., op. cit., p. 216-217.

32 Roberto Coroneo, «L'epigrafia greca medievale in Sardegna: a margine del libro di Andrè Guillou», Antonio M. Corda (ed.), Cultus splendore. Studi in onore di Giovanna Sotgiu. Senorbì: Edizioni Nuove Grafiche Puddu, 2003, p. 354.
} 
$(18 \times 139 \times 22$ e $18 \times 130 \times 22 \mathrm{~cm})$. Le lettere incise presentano un'altezza pari a $5 \mathrm{~cm}$. L'analisi visiva del manufatto ha consentito di mettere in luce alcuni danni subiti dai marmi, scheggiature ma anche abrasioni. ${ }^{33}$ All'estremità di entrambi i frammenti si riscontrano due decorazioni vegetali incorniciate da un riquadro. L'epigrafe viene citata dalla storiografia come quella di «Unuspiti e Sorica», dai nomi incisi nell'iscrizione. Il testo, preceduto da un segno di croce, riporta questa espressione formale: «Signore aiuta il tuo servo Unuspiti // e sua moglie Sorica così sia in umiltà». ${ }^{34}$ La tipologia dei marmi induce a pensare che si tratti di una coppia di mensole-architravi che originariamente facevano parte di un protiro mediobizantino di cui l'edificio fu dotato verso la metà del X secolo. ${ }^{35}$ L'ipotesi è supportata dal confronto tipologico effettuato con una coppia di elementi analoghi di protiro conservati nella basilica dei SS. Martiri a Cimitile, databile tra la fine dell'IX e gli inizi del X secolo. ${ }^{36}$ In uno dei suoi primi contributi, Antonio Taramelli ipotizzò che i due personaggi citati nell'epigrafe avessero uno stretto legame con la chiesa. La coppia poteva esservi stata sepolta oppure avere contribuito alla costruzione dell'edificio stesso, o a un suo restauro e abbellimento. ${ }^{37}$ Secondo Giandomenico Serra, invece, i due personaggi erano i fondatori o i patroni della chiesa di Santa Sofia, riconoscendo all'epigrafe un chiaro intento dedicatorio, ${ }^{38}$

${ }^{33}$ Roberto Coroneo, Scultura mediobizantina in..., op. cit., p. 216-217.

${ }^{34}$ Ibid., p. 216-217.

35 Ibid., p. 36.

${ }^{36}$ Ibid., p. 216-217.

${ }^{37}$ Antonio Taramelli, «Decimoputzu. Iscrizioni bizantine nella distrutta chiesa di S. Sofia», Accademia d'Italia, Notizie degli scavi di antichità comunicate alla $R$. Accademia dei Lincei per ordine di S. E. il ministro della pubb. Istruzione. Roma: coi tipi del Silviucci Supplementi, 1906, p. 132-135.

${ }^{38}$ Giandomenico Serra, «Nomi personali d'origine greco-bizantina fra i membri di famiglie giudicali o signorili del Medioevo sardo», Byzantion XIX (1949), p. 234. mentre per Letizia Pani Ermini si trattava dei committenti di un manufatto donato alla chiesa. ${ }^{39}$ Questo è l'unico caso nell'Isola in cui è esplicitata chiaramente la relazione parentale tra i personaggi citati. È infatti lo stesso epitaffio a precisare che Sorica è la moglie di Unuspiti. Si tratta con tutta probabilità di una coppia appartenente alla nobiltà del Giudicato di Cagliari, ma priva di incarichi od onorificenze, che, se presenti, sarebbero stati senza dubbio riportati nella dedica. ${ }^{40} \mathrm{I}$ marmi sono stati ritrovati contestualmente a un altro elemento lapideo, un'altra epigrafe che riporta i nomi dinastici di Torchitorio, Salusio e Ortzocor, due dei quali vengono definiti «nobilissimi arconti». ${ }^{41}$ Il marmo viene datato alla fine del $\mathrm{X}$ secolo. Pertanto, sebbene i due manufatti siano separati da all'incirca mezzo secolo, alcuni studiosi credono esista un legame parentale tra la coppia Unuspiti e Sorica e la famiglia arcontale. ${ }^{42}$ Roberto Coroneo identificava nel formulario e nel tono aulico la volontà di autocelebrazione dei coniugi. ${ }^{43}$ Lo stile utilizzato fa ricorso ampiamente a stilemi orientali, evidenti nella scelta del linguaggio. È singolare quanto questa classe politica, che da un lato cerca di affermare la propria emancipazione rispetto al potere orientale, dall'altro invece fa ricorso al legame ideale esistente sul piano culturale e religioso con Bisanzio, utilizzando schemi che sottendono all'Oriente stesso. ${ }^{44}$ I

\footnotetext{
${ }^{39}$ Letizia Pani Ermini, Museo Archeologico Nazionale di Cagliari. Catalogo dei materiali paleocristiani e altomedievali. Roma: Istituto poligrafico e zecca dello Stato, 1981, p. 52. Roberto Coroneo, Scultura mediobizantina in..., op. cit., p. 36.

40 Roberto Coroneo, Scultura mediobizantina in..., op. cit., p. 36. Antonio Taramelli, Di alcuni monumenti..., op. cit., p. 81 .

${ }^{41}$ Roberto Coroneo, Scultura mediobizantina in..., op. cit., p. 32-33. Marco Muresu, La moneta indicatore dell'assetto insediativo della Sardegna bizantina (secoli VI-XI). Perugia: Morlacchi, 2018, p. 353-354.

42 Antonio Taramelli, Di alcuni monumenti..., op. cit., p. 81.

${ }^{43}$ Roberto Coroneo, Scultura mediobizantina in..., op. cit., p. 216-217.

${ }^{44}$ Raimondo Turtas, Storia della Chiesa in Sardegna dalle origini al Duemila. Roma: Città Nuova, 1999, p.168.
} 
marmi epigrafici sarebbero parte integrante di un più ampio arredo liturgico a carattere dedicatorio che taluni studiosi mettono in relazione con le tombe a camera interrate rinvenute nel territorio tra Villasor e Decimoputzu, sostenendo la teoria di un possibile legame con l'arrivo di gruppi di cultura greco-orientale. ${ }^{45}$ Tutti questi elementi messi in relazione tra loro testimoniano una possibile continuità di vita nel territorio, abitato da gruppi i cui esponenti, di origine bizantina, costituirono una comunità con ruoli anche nell'esercito. Da questi si sarebbe sviluppata successivamente la civiltà giudicale. ${ }^{46}$ Senza dubbio le epigrafi, i possibili elementi d'arredo litico e le sepolture rimandano a un ambito

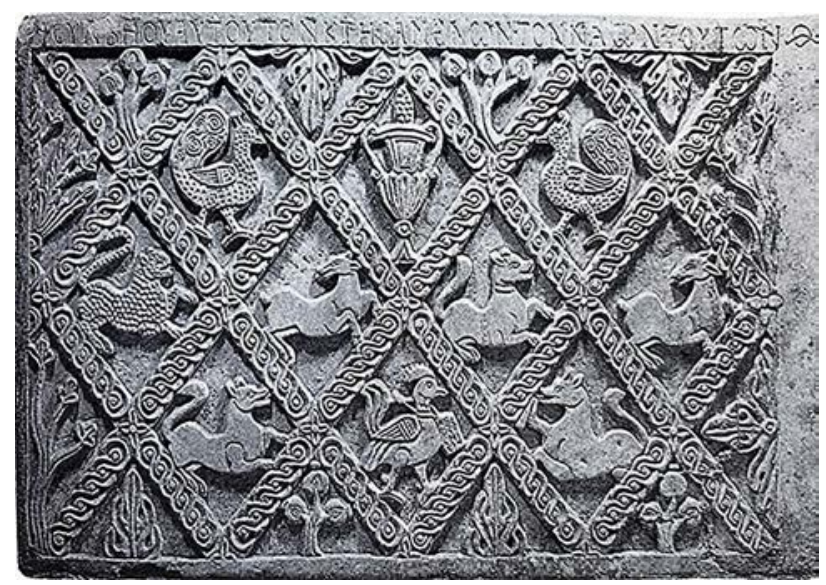

Figura 3. Napoli, epigrafe di Campulo e Costantina (da Coroneo 2000).

\footnotetext{
${ }^{45}$ Lucia Mura - Laura Soro, «I luoghi giudicali: dai documenti alle testimonianze archeologiche», Rossana Martorelli (ed.), Settecento Millecento. Storia, archeologia e arte nei "secoli bui" del Mediterraneo. Dalle fonti scritte, archeologiche ed artistiche alla ricostruzione della vicenda storica: La Sardegna laboratorio di esperienze culturali, vol. II. Cagliari: Scuola Sarda Editrice, 2013, p. 447-448.

${ }^{46}$ Ibid., p. 448. Olivetta Schena, «La Sardegna nel Mediterraneo bizantino (secoli VIII-XI): aspetti e problemi storici», Rossana Martorelli (ed.), Settecento Millecento. Storia, archeologia e arte nei "secoli bui" del Mediterraneo. Dalle fonti scritte, archeologiche ed artistiche alla ricostruzione della vicenda storica: La Sardegna laboratorio di esperienze culturali, vol. I. Cagliari: Scuola Sarda Editrice, 2013, p. 41-54.

${ }^{47}$ Roberto Coroneo, Scultura mediobizantina in..., op. cit., p. 23-30 e 37-43; Lucia Mura - Laura Soro, I lnoghi giudicali..., op. cit., p. 448.
}

sociale elevato. ${ }^{47}$ L'epigrafe di Unuspiti e Sorica può essere messa in relazione con un esemplare scultoreo ritrovato nell'ipogeo di Sant'Aspreno, presso Napoli. Si tratta di grandi plutei che nella fascia superiore riportano un epitaffio in lingua e caratteri greci, che restituisce i nomi di due coniugi, Campulo e Costantina ${ }^{48}$ (fig. 3-4). A partire dalla trascrizione in francese, effettuata da André Guillou, si ricava una traduzione in lingua italiana: «+ Ricordati Signore del tuo servo Campulo e di Costantina sua moglie che costruì questa chiesa». ${ }^{49}$ I marmi fanno parte di un più ampio arredo marmoreo mediobizantino composto da coppie di mensole-architrave e pilastri, oltre che da pilastrini e plutei, ritrovato nell'ambiente che

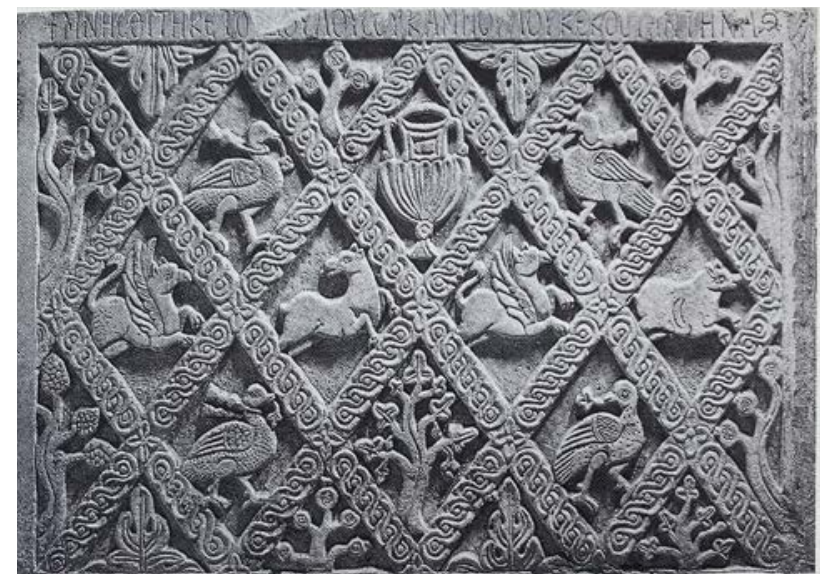

Figura 4. Napoli, epigrafe di Campulo e Costantina (da Coroneo 2000).

${ }^{48}$ Roberto Coroneo, Scultura mediobizantina in..., op. cit., p. 159-160.

49 André Guillou, Recueil des inscriptions grecques medievales d'Italie. Roma: Scuola Tipografica San Pio X, 1996, p.136.

${ }^{50}$ Roberto Coroneo, Scultura mediobizantina in..., op. cit., p.159. 
dà accesso all'oratorio dedicato al primo vescovo della città. L'impianto originario risale forse al VI secolo, su preesistenze di età romana. Nel 1859 la struttura fu inglobata nel palazzo della Borsa. ${ }^{50}$ Secondo le ricostruzioni effettuate dagli studiosi, i materiali litici ritrovati sono ascrivibili a un protiro simile a quello conservato nei SS. Martiri di Cimitile e a un recinto presbiteriale che, grazie all'epigrafe, possiamo attribuire alla committenza di una coppia dell'aristocrazia bizantina che lo fece produrre per la ormai scomparsa chiesa altomedievale. ${ }^{51} \mathrm{~L}$ 'iscrizione campana viene datata alla seconda metà del $\mathrm{X}$ secolo. ${ }^{52} \mathrm{Ad}$ accomunare questi materiali lapidei, provenienti da sponde opposte del Tirreno, non sono solo le circostanze di ritrovamento e il marcato riferimento a un contesto greco-orientale, quanto piuttosto anche e soprattutto il formulario. Entrambe le epigrafi, infatti, esprimono delle richieste dei committenti, riferibili forse più a un probabile ambito funerario che non dedicatorio. Entrambe le coppie di committenti, marito e consorte, non esplicitano una titolatura aristocratica, il che induce pertanto a pensare che i quattro fossero semplici membri dell'aristocrazia bizantina, esclusi da incarichi politici. Il formulario, simile ma non identico, fa probabilmente riferimento a tipologie in uso presso l'aristocrazia autoctona, il che spiegherebbe anche le differenze a livello linguistico. Da quanto emerso dalle riflessioni in merito al marmo epigrafico campano, i plutei si trovavano all'interno di una chiesa fatta erigere da Campulo e Costantina. Questo elemento sembra già sufficiente per inquadrare i manufatti in un contesto sociale di estremo rilievo. La loro raffinatezza si esprime attraverso la trama perlinata a nastro intrecciato che isola spazi romboidali entro i quali sono inserite figure animali $\mathrm{e}$ vegetali. ${ }^{53}$ La circolazione degli esemplari tra il

\footnotetext{
${ }^{51}$ Ibid., p.160. Guglielmo Cavallo, Le tipologie della..., op. cit., p. 494; Abbate Francesco, Storia dell'arte nell'Italia meridionale. Dai Longobardi agli Svevi. Roma: Donzelli editore, 1997 , p. 83.

${ }^{52}$ Roberto Coroneo, Scultura mediobizantina in..., op. cit., p. 159

${ }^{53}$ Ibid., p.182-184.
}

Mediterraneo occidentale e orientale è un dato di fatto che impone di considerare questi dettagli iconografici e stilistici come non riconducibili a un unico modello. ${ }^{54}$ È ampiamente comprovato dalla bibliografia precedente quanto questi stilemi siano ripresi dalle stoffe orientali, giunte in Italia tramite i mercanti, ma abbiano anche un sostrato locale che induce a volgere lo sguardo verso Ravenna e l'Esarcato bizantino. ${ }^{55}$ Sulla base di quanto detto, non si esclude evidentemente un'analisi comparativa tra la Sardegna e le realtà nord italiche che hanno mantenuto importanti legami con il mondo orientale.

\section{Epigrafe di Torcotorio e Getite}

Poco posteriore all'epigrafe di Unuspiti e Sorica è quella nota come di «Torcotorio e Getite» (fig. 5-6). Il marmo bianco fu ritrovato nel 1858, «nel disfarsi il pavimento della chiesuola di San Giovanni» ad Assemini. ${ }^{56} \mathrm{Nel} 1907$ il Taramelli la vide nella predella dell'altare del modesto oratorio di San Giovanni. ${ }^{57}$ Nel 2003 Roberto Coroneo la segnala ancora nella chiesa di San Giovanni Battista. ${ }^{58}$ L'epigrafe fino a poco tempo fa era composta di 5 frammenti, tuttavia, come si evince dall'analisi in situ, ora si contano 6 pezzi, a seguito di una frattura avvenuta in circostanze poche chiare. All'estremità destra risulta mutila. I frammenti combaciano, ma la rottura fra il secondo e terzo elemento ha provocato la perdita di parti minime dell'epigrafe. Le misure sono approssimativamente di $16 \times 177 \times 20 \mathrm{~cm}$, mentre

\footnotetext{
${ }^{54}$ Rossana Martorelli, «Su due pilastrini ad intreccio di epoca altomedievale dall'Iran», Rossana Martorelli (ed.), Itinerando. Senza Confini dalla preistoria a oggi. Studi in ricordo di Roberto Coroneo, vol. 1.2. Perugia: Morlacchi Editore, 2015, p. 531-534.

${ }_{55}$ Roberto Coroneo, Scultura mediobizantina in..., op. cit., p.182-184.

56 Pietro Martini, «Iscrizione greca di Assemini», Bullettino Archeologico Sardo VII (1861), p. 150.

${ }^{57}$ Antonio Taramelli, aDi alcuni monumenti..., op. cit., p. 73.

${ }^{58}$ Roberto Coroneo, L'epigrafia greca medievale..., op. cit., p. 350.
} 


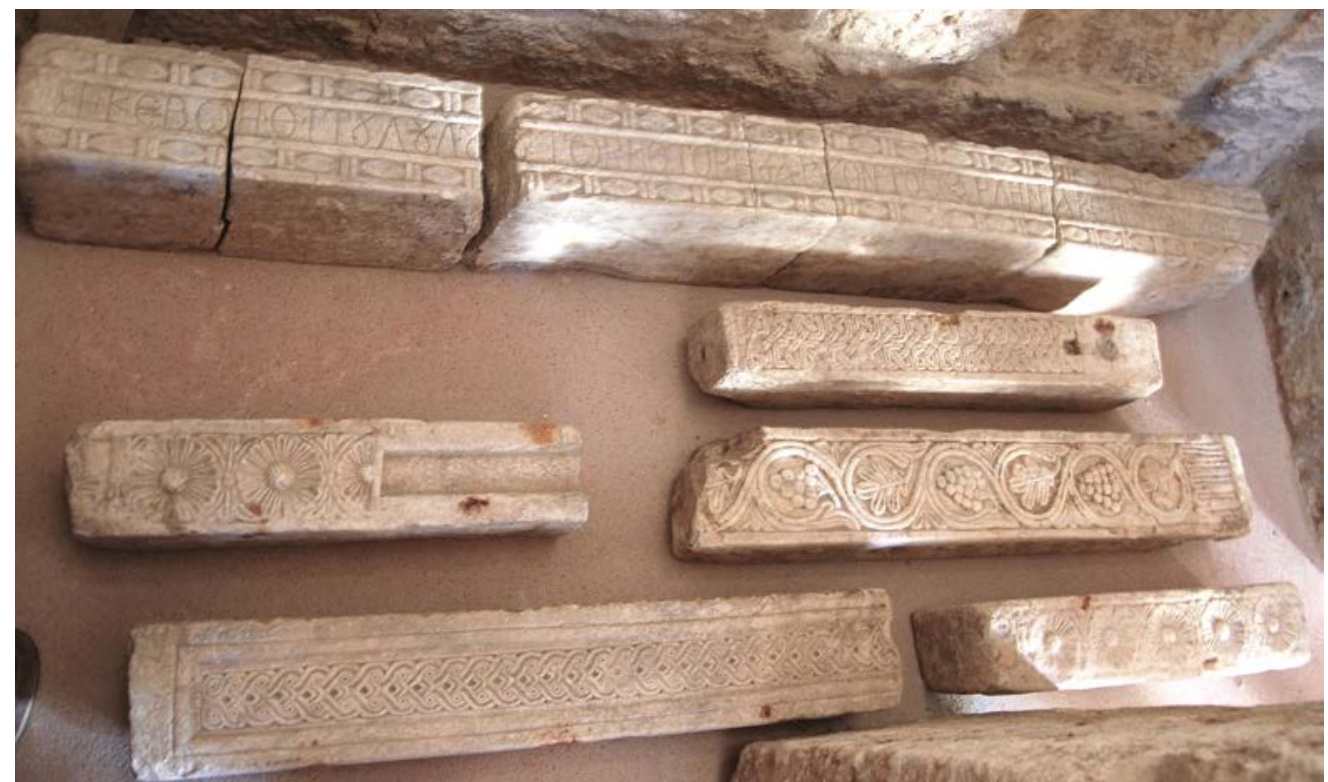

Figura 5. Assemini, epigrafe di Torcotorio e Getite (Andrea Pala).

Figura 6. Assemini, epigrafe di Torcotorio e Getite (Andrea Pala).

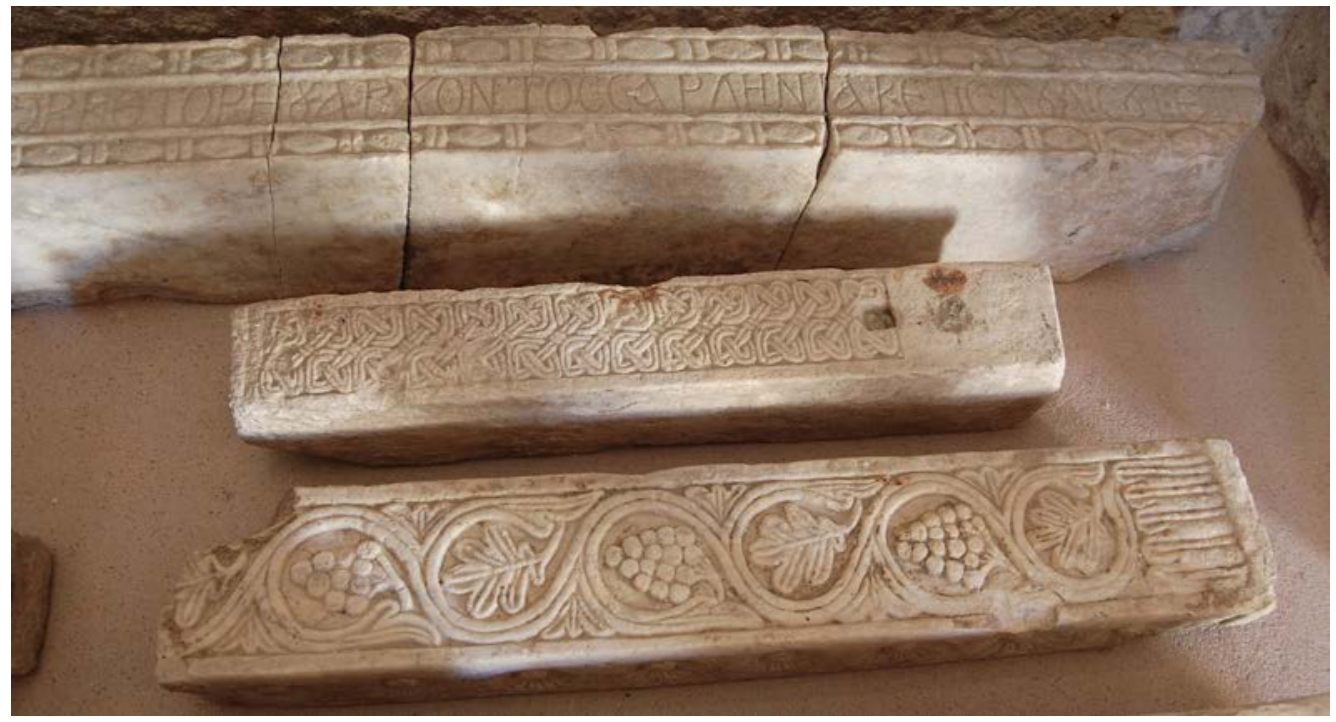

l'altezza delle lettere incise è di $\mathrm{cm} 4,5 .{ }^{59}$ L'ornato a fuseruole che delimita l'epitaffio trova riscontri simili in gran parte della produzione plastica del Sud Italia. Tuttavia, il confronto maggiormente stringente si ha con le fuseruole che riquadrano i pilastrini di protiro di Sant'Aspreno di Napoli, ascrivibili alla seconda metà del $\mathrm{X}$ secolo. ${ }^{60}$ Anche in questo caso, l'iscrizione è introdotta

\footnotetext{
${ }^{59}$ Roberto Coroneo, Scultura mediobizantina in..., op. cit., p. 208.

${ }^{60}$ Ibid., p.159-166.
}

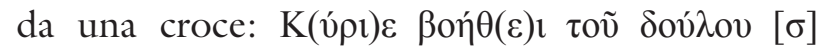

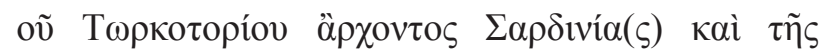

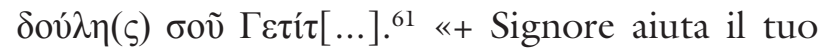
servo Torcotorio, arconte di Sardegna, e la tua serva Getite». ${ }^{62}$ I caratteri epigrafici e il partito

${ }^{61}$ Marco Muresu, La moneta indicatore..., op. cit., p. 353.

62 Simonetta Angiolillo, «La Sardegna romana e altomedievale. Catalogo», Simonetta Angiolillo et alii (coords.), Corpora delle antichità della Sardegna. La Sardegna romana e altomedievale. Storia e materiali. Sassari: Carlo Delfino Editore, 2017 , p. 448. 
compositivo e ornamentale sembrano essere simili a quelli dell'iscrizione di Torcotorio, Salusio e Ortzocor, ritrovata presso la già citata chiesa distrutta di San Sofia. ${ }^{63}$ L'originaria funzione del marmo potrebbe essere quella di un architrave di recinto presbiterale, tanto che gli studiosi ipotizzano un'associazione di questi materiali litici con il resto dell'arredo marmoreo ritrovato nel San Giovanni di Assemini e composto da pilastrini e plutei. ${ }^{64}$ I quesiti inerenti a questi frammenti lapidei sono vari. Il ritrovamento presso la suddetta chiesa non può considerarsi automaticamente una prova dell'originaria appartenenza del materiale all'arredo liturgico della stessa. L'alta qualità in questo caso, come nel precedente, indurrebbe a riflettere sulla possibile funzione di questi marmi. Il formulario è stato individuato, su riflessioni linguistiche, come appartenente alla categoria delle epigrafi dedicatorie. ${ }^{65}$ I personaggi citati sono segnalati dalla bibliografia precedente come marito e moglie. Questa definizione non si basa sulla lettura dell'epigrafe, nella quale peraltro non è specificato il grado di parentela che li unisce, ma su riflessioni generate attorno al ridotto materiale storico al quale si può avere accesso. Il Martini partì dal dato storico che nel 1107 a offrire il San Giovanni di Assemini al San Lorenzo di Genova fu un tale Mariano-Torcotorio II. ${ }^{66}$ In virtù di questo, lo studioso fece coincidere il personaggio dell'epigrafe e suddetto giudice con la figura del donatore dell'edificio ecclesiastico. ${ }^{67}$ Tale identificazione non coincide con un altro dato storicamente accertato, ossia che il MarianoTorcotorio II, proposto dal Martini, aveva una moglie che si chiamava Preziosa e non Getite. ${ }^{68}$

\footnotetext{
${ }^{63}$ Roberto Coroneo, L'epigrafia greca medievale..., op. cit., p. 354.

${ }^{64}$ Roberto Coroneo, Scultura mediobizantina in..., op. cit., p. 60 .

${ }_{65}$ Ibid., p. 208.

${ }^{66}$ Pietro Martini, Iscrizione greca di..., op. cit., p. 149.

67 Ibid., p. 150.

${ }^{68}$ Lindsay Leonard Brook - Francesco Cesare Casula et alii (coords.), Genealogie medioevali di Sardegna. Cagliari, Sassari: Due D Editrice mediterranea, 1984, p.177.
}

Un'ulteriore problematica che si palesa è, secondo il Taramelli, quella riguardante la dicitura «arconte di Sardegna», titolo con il quale si definisce Torcotorio. Sembrerebbe che la menzione si riferisca a un dominio ancora indiviso dell'Isola, incongruente con la situazione effettiva del XII secolo, epoca nella quale vive Mariano-Torcotorio II. ${ }^{69}$ Altri studiosi hanno invece proposto un'identificazione tra il Torcotorio dell'epigrafe di Assemini e il Torcotorio «protospatario imperiale» dell'iscrizione rinvenuta presso la chiesa di Santa Sofia. ${ }^{70}$ Di più complessa riconoscibilità è la figura femminile. Il nome è anzitutto tronco nella parte finale. Secondo Giandomenico Serra la parola viene da Getis/Getida. Si tratterebbe quindi di un nome personale greco. ${ }^{71}$ Questa nobildonna sarebbe la stessa che lasciò in eredità a Mariano Torcotorio II l'isola di Sant'Antioco prima che questa fosse donata al Santo nel 1124 come patrimonio della dinastia cagliaritana dei LaconGunale. $^{72}$ I personaggi citati per nome sono corredati da titoli imperiali di matrice bizantina dei quali, è ipotizzabile, siano stati insigniti dal potere politico orientale. Sulla dinamica dei rapporti intessuti tra la Sardegna e Bisanzio gli studiosi si sono confrontati a lungo e questi titoli onorifici, o presunti tali, hanno contribuito ad ampliare il contesto della discussione. ${ }^{73}$ Tra le fonti orientali che citano la Sardegna il De cerimoniis Aulae Byzantinae, attribuito a Costantino VII Porfirogenito, si configura come un ottimo strumento di riflessione sul tema. Nello specifico, l'Isola è citata tra gli Stati esteri le cui delegazioni si presentano davanti all'Imperatore.

\footnotetext{
${ }^{69}$ Antonio Taramelli, Di alcuni monumenti..., op. cit., p. 75.

${ }^{70}$ Roberto Coroneo, Scultura mediobizantina in..., op. cit., p. 32 .

${ }^{71}$ Giandomenico Serra, Nomi personali d'origine..., op. cit., p. 223-245

${ }_{72}$ Roberto Coroneo, Scultura mediobizantina in..., op. cit., p. 32.

${ }^{73}$ Marco Muresu, La moneta indicatore..., op. cit., p. $353-$ 356.
} 
Tutti i Paesi, riportati in uno scrupoloso ordine, ${ }^{74}$ sembrano gravitare nell'orbita di Costantinopoli, anche se caratterizzati da una propria identità politica spesso tendente all'indipendenza. ${ }^{75}$ Sulla base di quanto detto, alcuni studiosi hanno ipotizzato che Bisanzio cercasse di tenere $\mathrm{i}$ suddetti Paesi sotto la propria sfera d'influenza, donando ai propri esponenti di spicco titoli dignitari. $^{76} \mathrm{~A}$ riprova di ciò, si noti come la maggior parte delle realtà politiche del Sud Italia «sono accumunate da una base culturale di tipo orientale, riconoscibile nelle titolature, nelle prassi documentarie e in un richiamo costante [...] al peso ideologico di Bisanzio come fonte di legittimità del proprio potere». ${ }^{77} \mathrm{La}$ coincidenza terminologica delle cariche di iudex e $d u x$, tra il IX e il X secolo, in Sardegna e nei territori di Amalfi e Napoli, comporta un'interessante sosta riflessiva sulla natura di questi titoli che alcuni studiosi hanno definito «titoli di funzione». ${ }^{78} \grave{\mathrm{E}}$ inoltre auspicabile che questi, se correttamente interpretati, possano dare alcuni suggerimenti in merito ai personaggi che ne sono insigniti. Nel caso isolano si tratta certamente di appartenenti all'alta nobiltà che si esprimono tramite una committenza aulica denotata dall'alto livello tecnico dei materiali. ${ }^{79}$ I giudici di Cagliari si considerarono sempre i legittimi discendenti dell'arconte di Sardegna bizantino. Ne sono testimonianza la produzione di documenti ancora in caratteri greci o l'utilizzo in alternanza dinastica dei nomi Torchitorio e Salusio, che sono gli stessi presenti nelle epigrafi medioelleniche. ${ }^{80}$

\footnotetext{
${ }^{74}$ Agostino Pertusi, «Insegne del potere sovrano e delegato a Bisanzio e nei paesi di influenza bizantina», Centro italiano di studi sull'alto Medioevo, Simboli e simbologia nell'alto medioevo (3-9 aprile 1975), vol. II. Spoleto: Centro italiano di studi sull'alto Medioevo, 1976, p. 481-564 e 557558 .

${ }^{75}$ Marco Muresu, La moneta indicatore..., op. cit., p. 346; Luciano Gallinari, Il giudicato di..., op. cit., p. 152. Raimondo Turtas, Storia della Chiesa..., op. cit., p. 167.

${ }^{76}$ Marco Muresu, La moneta indicatore..., op. cit., p. 347; Agostino Pertusi, Insegne del potere..., op. cit., p. 560-561.

${ }_{77}$ Marco Muresu, La moneta indicatore..., op. cit., p. 350.

78 Ibid., p. 353.

79 Roberto Coroneo, Scultura mediobizantina in..., op. cit., p. 8.

${ }^{80}$ Luciano Gallinari, Il giudicato di..., op. cit., p.156.
}

In quest'ottica, dunque, è il Giudicato di Cagliari ad avere intessuto i migliori rapporti con l'Oriente sin dal principio, quando, tra il 533 e il 534, la Sardegna fu annessa ai territori dell'Impero romano d'Oriente, divenendo una delle sette province africane dipendente da un Prefetto del Pretorio che risiedeva a Cartagine, ma avente un proprio praeses e un proprio $d u x$ sul territorio. ${ }^{81}$ I legami, come evidenziato in precedenza, si sono mantenuti anche successivamente in qualità di erede dell'autorità antica. Ciò è testimoniato anche dalla pluralità di influenze artistiche che si registrano nell'Isola già prima del IX secolo e che, prudentemente, individuano Cagliari molto più vicina all'Oriente, rispetto a una Turris Libisonis forse più vicina agli ambienti ecclesiastici latini. ${ }^{82}$

\section{Le epigrafi di Nispella}

Le riflessioni portate avanti in questa breve digressione storica sono condivisibili anche nel caso di un'altra iscrizione ritrovata nel Giudicato di Cagliari, ossia quella che riporta i nomi di Torcotorio e Salusio e di Nispella (fig. 7). I tre frammenti che la compongono furono ritrovati murati nell'altare maggiore della Basilica di Sant'Antioco, nell'omonima Isola. ${ }^{83}$ In questo luogo li vide il Taramelli nel 1907, anticipando già l'ipotesi secondo la quale quella non sarebbe stata la loro collocazione originaria. I frammenti, piuttosto, sarebbero stati parte integrante di un grande monumento intorno al quale correva l'iscrizione. ${ }^{84}$ I resti, dunque, furono rimossi dall'altare nel 1966 per essere depositati sulla mensa del sarcofago-altare nella cripta. ${ }^{85}$ I danni

\footnotetext{
${ }^{81}$ Alberto Boscolo, La Sardegna bizantina e altogiudicale. Sassari: Chiarella, 1978, p. 33.

82 Roberto Coroneo, La cultura artistica..., op. cit., p. 105; Valeria Carta, I marmi mediobizantini..., op. cit., p. 345 .

${ }^{83}$ Roberto Coroneo, L'epigrafia greca medievale..., op. cit., p. 362.

${ }^{84}$ Antonio Taramelli, Di alcuni monumenti..., op. cit., p. 83.

${ }^{85}$ Roberto Coroneo, Scultura mediobizantina in..., op. cit., p. 87.
} 
che ha subito il marmo hanno determinato la perdita della parte iniziale e intermedia dello scritto. Le misure, rilevate dal Roberto Coroneo, sono pari a $20 \times 211 \times 23 \mathrm{~cm}$, con un'altezza delle lettere incise di $9 \mathrm{~cm}$. Originariamente, i frammenti potrebbero avere fatto parte di un architrave di recinto presbiterale. ${ }^{86}$ L'epigrafe si conclude con una foglia d'edera incisa e riporta queste parole:

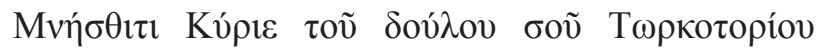

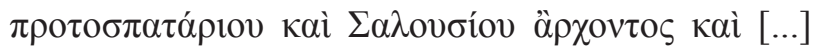

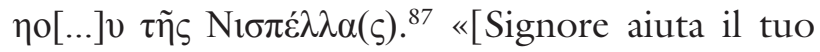
servo/i tuoi servi] Torcotorio protospatario e Salusio arconte e $[\ldots]$ di Nispella». ${ }^{88}$ Le personalità riportate sono state individuate come la coppia giudicale in carica, Torcotorio e Nispella, al cui governo era associato Salusio. ${ }^{89}$ Questa lettura nasce dall'ipotesi che il protospatario altri non sia che il giudice di Cagliari, ricordato nella Vita di San Giorgio di Suelli e sposato con Nispella. ${ }^{90}$ Salusio, indicato come "arconte», sarebbe invece il successore di Torchitorio, per molti il figlio stesso. ${ }^{91}$ Secondo la sintesi riportata da Roberto Coroneo nell'epigrafe di Sant'Antioco, la coppia reale sarebbe quella che donò la Villa di Suelli e di Simieri (Selegas) a San Giorgio. Il fatto è riportato nella Legenda Sancti Georgi ed è accennato nell'atto di Benedetta Lacon Massa al vescovo del 1215. Nel primo caso la giudicessa è detta Sinispella, nel secondo Nispella. Se ne deduce che se sono contemporanei a San Giorgio di Suelli vescovo, sono vissuti tra la fine del X e i primi decenni dell'XI secolo, post $1015 .^{92}$ Il nome femminile compare in due epigrafi successive, datate ambedue alla prima metà dell'XI secolo ed entrambe ritrovate ad Assemini. Per gli studiosi apparterrebbero alla committenza dello stesso personaggio citato nell'epigrafe di Sant'Antioco e nei documenti pergamenacei del XII-XIII secolo. ${ }^{93}$ La prima epigrafe, che già agli inizi del nuovo millennio risultava dispersa, era stata ritrovata nella chiesa parrocchiale di San Pietro ad Assemini nel 1919. Le notizie che ci vengono riportate descrivono

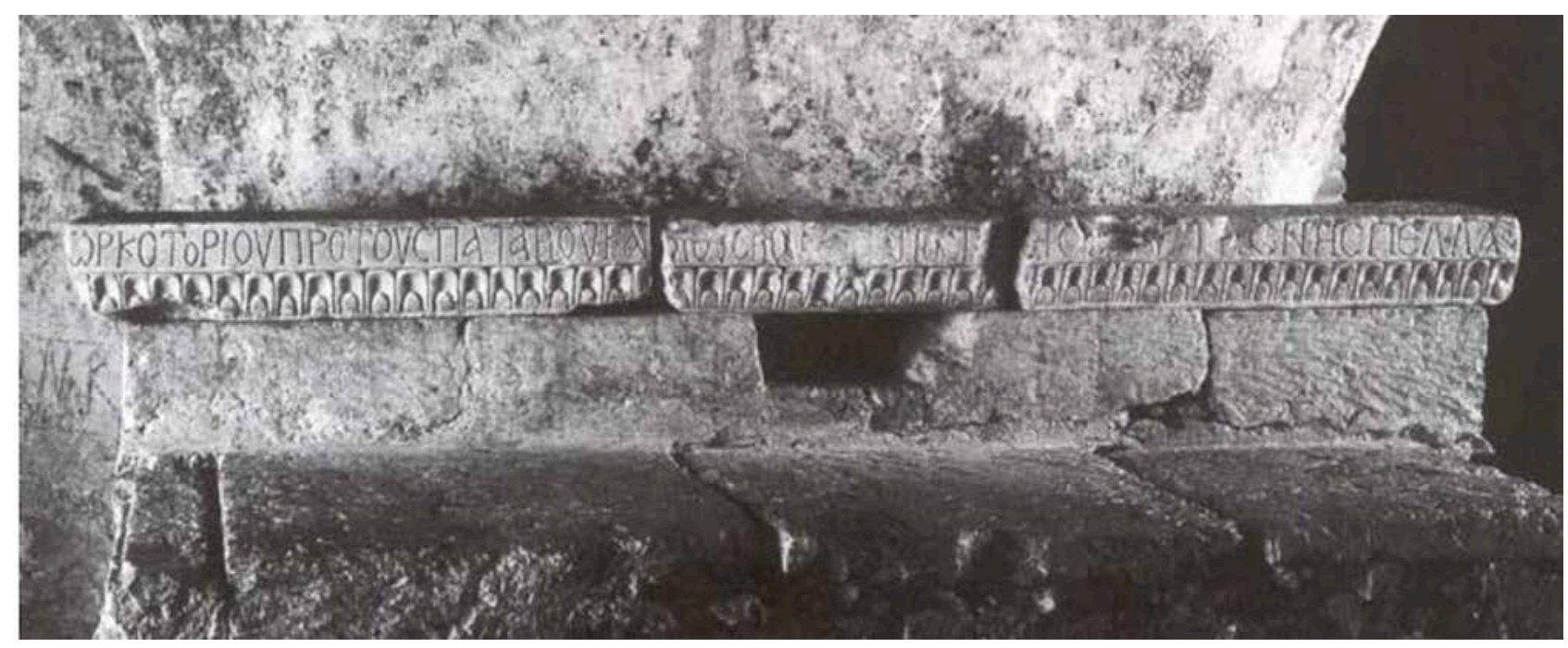

Figura 7. Sant'Antioco, epigrafe di Torcotorio, Salusio e Nispella (da Curreli 2012).
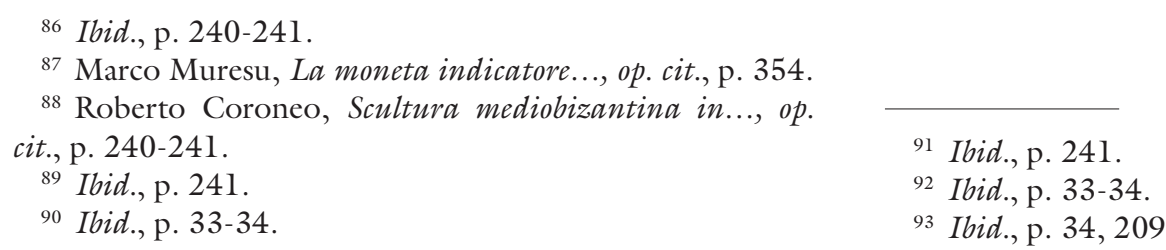
una lastra in forma di parallelepipedo in marmo bianco, mutila all'estremità destra. L'iscrizione in lingua e grafia greca, introdotta da croce e incisa su 4 linee, recita così: I. «+ Io Nispella [] II-IV []». ${ }^{94}$ Non è stata proposta ancora una lettura completa, anche se le poche lettere incise rievocano la figura della moglie del giudice già citata a Sant'Antioco. Appare impossibile, al momento, individuare la funzione di questo marmo, considerate le sue esigue dimensioni. ${ }^{95}$ L'altra epigrafe proviene ugualmente dalla chiesa di San Pietro, dove il frammento fu reimpiegato come soglia (fig. 8). A seguito delle abrasioni riportate è andata perduta la parte superiore della prima linea. Almeno dagli inizi del Novecento fu spostata presso l'edificio dedicato a San Giovanni del medesimo centro abitato. ${ }^{96}$ L'iscrizione, introdotta da croce greca e divisa su tre linee, recita così: " + Nel nome del Padre, del Figlio e dello Spirito Santo, io Nispella [] di Ocote [] II. Dei Santi corifei apostoli Pietro e Paolo e di San Giovanni battista [] III. Vergine e martire Barbara affinché per loro intercessione mi conceda il Signore la remissione []». ${ }^{97} \mathrm{Se}$ ne conserva memoria grazie alla trascrizione di Michele Plazza. ${ }^{98} \mathrm{Il}$ formulario induce a credere si tratti di un'epigrafe dedicatoria che celebra la committenza della consorte del giudice. L'originaria funzione del marmo è incerta. E troppo pesante per essere un architrave di recinto presbiterale o di un portale. Forse era un frontale d'altare. ${ }^{99}$ Secondo il Taramelli e il Solmi, l'iscrizione è la prova del fatto che Nispella fu la fondatrice o donatrice della chiesa, o anche degli altari, dei SS. Pietro e Paolo, San Giovanni Battista e Santa Barbara. Tale teoria era abbracciata anche dallo Spano. ${ }^{100}$ Tuttavia l'ipotesi che Nispella sia la fondatrice del San Giovanni di Assemini è messa in dubbio da Coroneo perché la chiesa doveva già esistere se Torcotorio e Getite la dotano di arredi. Pertanto, forse Nispella ha fondato la chiesa dei Santi Apostoli e ha apportato donazioni alle strutture già esistenti, con arredi liturgici che potevano essere un altare o il portale. ${ }^{101}$ La lettura di Nispella, in quanto «figlia di Ocote», apre un ulteriore quesito sull'identità di questa figura. ${ }^{102}$

\section{Osservazioni conclusive}

Il panorama appena accennato sulle epigrafi mediobizantine in Sardegna sfortunatamente apre più interrogativi che non elementi utili a una chiarificazione. Gli ultimi due materiali lapidei citati sono probabilmente gli unici indizi di una forte autonomia delle figure femminili. Infatti, se negli altri documenti epigrafici le donne consorti sono associate al potere maschile, in questo caso è la stessa Nispella a rivolgere una richiesta di intercessione per la remissione dei peccati e, verosimilmente, lo fa attraverso una commissione

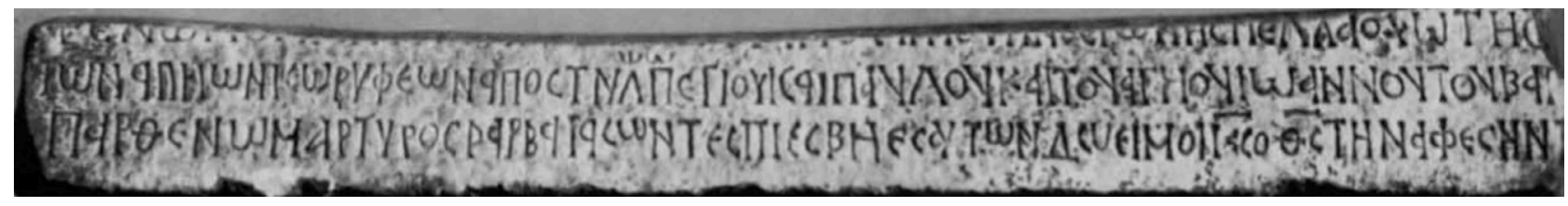

Figura 8. Assemini, epigrafe di Nispella (da Delogu 1988).

\footnotetext{
${ }^{94}$ Ibid., p. 209.

95 Ibid., p. 209.

96 Roberto Coroneo, L'epigrafia greca medievale..., op.cit., p. 348 .

97 Roberto Coroneo, Scultura mediobizantina in..., op. cit., p. 208; Simonetta Angiolillo, La Sardegna romana..., op. cit., p. 449.

${ }_{98}$ Antonio Taramelli, Di alcuni monumenti..., op. cit., p. 75 .
}

\footnotetext{
99 Roberto Coroneo, Scultura mediobizantina in..., op. cit., p. 208.

${ }^{100}$ Ibid., p. 34

${ }^{101}$ Ibid., p. 34

${ }^{102}$ Michele Orrù, Le fonti greche..., op. cit., p. 22-23.
} 
artistica. Prendendo in esame i luoghi dai quali questi materiali giungono, si può confermare quanto evidenziato in occasione dello studio di alcuni manufatti riconducibili al medesimo arco cronologico e reimpiegati su chiese posteriori. ${ }^{103}$ I centri abitati della provincia di Cagliari restituiscono un numero estremamente alto di frammenti epigrafici e figurativi che testimoniano chiaramente una committenza attiva, non solo nella capitale, ma anche nell'entroterra. Quanto già notato nel precedente studio emerge anche in questo contesto. I frammenti sembrano pervenire in modo cospicuo da centri posti sulla CaralibusTurrem, detta anche «via dei greci». ${ }^{104}$ Le epigrafi

${ }^{103}$ Valeria Carta, I marmi mediobizantini..., op. cit., p. 341-354.

${ }^{104}$ Ibid., p. 342. medioelleniche sarde, dunque, si inquadrano in un contesto storico peculiare, che ancora attende una puntuale codificazione. In tempi recenti alcuni studiosi hanno concentrato la loro attenzione su elementi epigrafici passati quasi inosservati che perpetuano l'uso della lingua greca all'interno dei luoghi di culto, ma che sono stati tuttavia segnati da numerosi interventi in fasi successive e la cui cronologia non è ancora del tutto chiara. ${ }^{105}$ L'obiettivo sarà forse raggiungibile grazie allo sforzo congiunto di studiosi afferenti a diverse discipline che sappiano guardare al problema con occhio critico e comparativo rispetto ad altre realtà europee. ${ }^{106}$

\footnotetext{
${ }^{105}$ Alessandro Teatini, «Minima Nova epigraphica dalla catacomba di Sant'Antioco", Quaderni. Rivista di Archeologia 28 (2017), p. 344.

${ }^{106}$ Marco Muresu, La moneta indicatore..., op. cit., p. 358359
} 